SVEPM 2015 - Controlling disease across species using emerging techniques in epidemiology and economics applied to animal health, Society of Veterinary Epidemiology and Preventive Medicine conference, Ghent, Belgium, 25-27 March 2015

Nielsen, Liza Rosenbaum; Borne, Bart H.P. van den; Brodbelt, David; Christley, Rob M.; McIntyre, Marie

Published in:

Preventive Veterinary Medicine

DOI:

10.1016/j.prevetmed.2015.10.021

Publication date:

2015

Document license:

Other

Citation for published version (APA):

Nielsen, L. R., Borne, B. H. P. V. D., Brodbelt, D., Christley, R. M., \& Mclntyre, M. (2015). SVEPM $2015-$

Controlling disease across species using emerging techniques in epidemiology and economics applied to animal health, Society of Veterinary Epidemiology and Preventive Medicine conference, Ghent, Belgium, 25-27 March 2015. Preventive Veterinary Medicine, 122(4), 379-380. https://doi.org/10.1016/j.prevetmed.2015.10.021 


\section{SVEPM 2015-Controlling disease across species using emerging techniques in epidemiology and economics applied to animal health, Society of Veterinary Epidemiology and Preventive Medicine conference Ghent, Belgium 25-27 March 2015}

At the 2015 Annual Meeting of the Society for Veterinary Epidemiology and Preventive Medicine (SVEPM) in Ghent, Belgium, several studies were presented which applied new and mixed methodologies in the search for explanations and solutions to prevent disease in animals and humans. Among the studies presented, the eight published in this special edition of Preventive Veterinary Medicine demonstrate both the high quality science presented at this conference and, as pointed out by one of the keynote speakers, that preventive veterinary medicine is an important contributor to sustainable animal production today.

The papers focus on a wide spectrum of topics and approaches, including both qualitative and quantitative methods investigating opinions and drivers for proactive animal health planning in sheep, a participatory method for surveillance evaluation, sample size considerations for livestock movement network data, spatiotemporal analyses combined with cost-benefit considerations of a tick-borne disease, economic impact of subclinical disease in cattle, state-of-the-art epidemiological analyses of antimicrobial resistance trends in multiple species and analysis of register data on dog diseases.

In the first paper, Bellet et al. used qualitative interviews with 12 veterinarians in central, south-west and the north of England and Wales to understand veterinarians' opinions on preventative advice and drivers for current services to sheep farmers. This was followed up by quantitative analysis of answers from 147 veterinarians who responded to a postal questionnaire of belief statements. Three main factors were identified: motivation for proactiveness, perceived capability to offer preventative services and perceived opportunity to deliver these services. These factors were associated with the proportion of time spent in an advisory role with sheep clients. Such insight into the nature and drivers of veterinarians' behaviour and beliefs can help in designing efficient strategies aimed at promoting proactive health services offered by veterinarians on sheep farms.

Calba et al. illustrated how qualitative research methods can be used to improve surveillance systems. They used eight individual semi-structured interviews and three focus groups of sixteen essential stakeholders, conducted using participatory approaches, to evaluate two attributes of surveillance for African swine fever on the island of Corsica: the acceptability of the surveillance system and its non-monetary benefits. The analytic methods included diagramming and scoring tools used to assess the different elements of acceptability, and a contingent valuation method, associated with proportional piling, used to assess the value of sanitary information. Results highlighted a moderate acceptability of the system for farmers and hunters and a high acceptability for other representatives (e.g. private veterinarians and local laboratories).

In the third paper, Pfeiffer et al. focused on another aspect of surveillance and control of disease in livestock, namely social network analysis. The effect of using animal movements between farms to identify high-risk premises may be affected by having incomplete networks, which may occur when sampling from the network. In the paper, a simulation algorithm is presented that provides an estimate of required sampling proportions based on predicted network size, density and degree value distribution. It can be used to tailor study designs of known precision for investigation of livestock movement networks and their impact on disease dissemination within populations.

In the fourth paper, Shaw et al. present results of an investigation and mapping of the cost-benefits from bovine trypanosomosis control and elimination in East Africa. The authors compared five intervention techniques: trypanocides, targets, insecticide-treated cattle, aerial spraying and the release of sterile male tsetse flies. The results indicate that no single technique or strategy is universally profitable. Furthermore, if the burden of human African trypanosomosis were factored in, the benefit-cost ratios of some of the low-return areas considerably increased. Comparatively, elimination strategies gave rise to higher benefit-cost ratios than continuous control. However, the costs calculated for elimination assume problem-free, large scale operations, and they rest on the outputs of entomological models that are difficult to validate in the field.

Raboisson et al. also used economics applied to animal health in their investigation of the total costs of subclinical ketosis, a common metabolic disorder in dairy cows. Information from a previous meta-analysis on production and reproduction changes and health disorders associated with subclinical ketosis was used in a stochastic cost estimation approach. It was found that the total cost of 
complex health disorders is likely to be substantially overestimated when calculations use raw results from the literature or point estimates as opposed to input distributions such as those from the meta-analysis. Furthermore, the authors argued that the applied approach would be easily and practically used by decision makers in the field.

In the sixth paper, Pugh et al. described the distribution of canine lifestyle and health parameters from a research initiative ('Dogslife') that collects data directly from dog owners of Kennel Club-registered Labrador Retrievers in the United Kingdom. A selection of data from Dogslife regarding the height, weight and lifestyle of 4307 Labrador Retrievers up to four years of age is reported in the paper. In the future these data will be used for more detailed analysis of associations between lifestyle and health, and potential options for prevention of canine disorders.

In another study on dogs, Mattin et al. estimated survival times of 237 dogs with electronic patient records of degenerative mitral valve disease (DMVD) in a retrospective cohort study of dogs attending 93 primary-care veterinary practices in England between January 2010 and December 2011. The median survival time for allcause mortality was 2-3 years after disease detection for DMVD cases. The mean age at which a heart murmur was first recorded in possible cases was close to 10 years. In multivariable survival analysis, Cavalier King Charles Spaniels and other purebreds had higher hazards of death than crossbreds. Dogs weighing $\geq 20 \mathrm{~kg}$ and older dogs had an increased hazard of death compared with those weighing less or younger dogs. Solid survival studies such as this one provide practicing veterinarians with good prognostic values for specific diseases.

In the last paper, Hanon et al. performed a temporal trend analysis on antimicrobial resistance data collected over 4 consecutive years (2011-2014) in the official Belgian antimicrobial resistance monitoring programme. During this period, antimicrobial use in livestock was generally reduced. The resistance prevalence remained high $(>50 \%$ ) during the study period for sev- eral antimicrobial classes and species. State-of-the-art statistical analyses identified decreasing temporal trends for several of the tested antimicrobials in several livestock categories. However, a significant increasing trend was observed in the prevalence of resistance to ciprofloxacin in chickens. Such outputs can be used as objective evidence to evaluate the possible efficacy of measures taken by animal health authorities and stakeholders to limit antimicrobial resistance issues that pose risks to both humans and animals.

This special edition of Preventive Veterinary Medicine provides an overview of current, advanced veterinary epidemiological research being conducted all over the world for the benefit of animal and human health. We acknowledge the work done by the members of the local organising committee in Belgium, which enabled such a varied selection of work to be presented in a superb location. The reviewers of the papers for this special issue are thanked for their careful peer-reviews. Also, members of the executive committee of The Society for Veterinary Epidemiology and Preventive Medicine deserve special thanks for their timely and thorough work during the selection and editing of both the 21 conference proceedings and the eight papers contained within this special edition.

Liza Rosenbaum Nielsen* Bart H.P. van den Borne David Brodbelt Rob M. Christley Marie McIntyre

The Society for Veterinary Epidemiology and Preventive Medicine

* Corresponding author at: University of Copenhagen, Denmark. E-mail address: liza@sund.ku.dk (L.R. Nielsen) 